\title{
Split-ring resonator: A new detector in liquid chromatography
}

\author{
Kirsten J. Dehning, Moritz Hitzemann, Sven Fisahn, Stefan Zimmermann \\ Leibniz University Hannover, Institute of Electrical Engineering and Measurement Technology, \\ Appelstr. 9A, 30167 Hannover, Deutschland \\ Contact:dehning@geml.uni-hannover.de
}

\section{Introduction}

There is a wide variety of applications that use liquid chromatography (LC) as a physical separation technique for liquid samples e.g in food or drug analysis [1,2]. The LC is often combined with optical detectors that detect the eluting substances according to their fluorescence, absorption and refraction charateristics. Some substances have to go through a time consuming and elaborate sample preparation process so that they can be detected by such optical detectors. Other detectors use a different charateristic for detection, e.g. mass spectrometers that allow the orthogonal identification of analytes. However, major disadvantages are high purchasing and operating costs and the considerable intrumental effort, besides the destruction of the sample due to the required ionization [3]. This spikes the interest in new nondestructive and simple detectors. Here, we present a detector that is based on a split-ring resonator (SRR) and uses the relative permitvity instead of optical properties. Split-ring resonators are simple and very sensitive sensors. Gehl et. al introduced a first split-ring resonator as a possible detector for liquid chromatography [4].

Split-ring resonators mostly consist of a simple printed curcuit board that has a ring structure and a strip line. The electromagnetic wave is coupled into the system via the strip line which has an impedance of $50 \Omega$ adapted to the measurement system. The transmission from the input of the strip line to its output varies in dependency of the energy that is coupled into the ring structure. The resonance frequency of the split-ring resonator can be determined by measuring the transmission through the strip line for example using the scattering coefficient $S_{21}$ or by directly measuring the output power.

The ring is like an RLC-resonator so the resonance frequency depends on the geometry of the ring and the relative permittivity in the split that acts as a capacitor. The resonance frequency of such circuit can be calculated via (1) $[5,6]$ :

$$
f_{\text {res }}=\frac{1}{2 \pi \cdot \sqrt{L C}}
$$

At the resonance frequency the inductive coupling of the wave into the ring has a maximum. The sample with a complex permittivity $\varepsilon_{r}=\varepsilon_{r}^{\prime}-j \varepsilon_{r}^{\prime \prime}$ will be placed at the split. Thus, the measurement principle is based on the change of the split capacitor dominated by the real part of the relative sample permittivity $\varepsilon_{r}^{\prime}$ causing a change of the resonance frequency[4].

The transmission line theory provides another link between the real part of the sample permittivity and the resonance frequency. With the assumption that the ring is a dipole antenna with capacitive prolongation the following equation results:

$$
\begin{aligned}
& \frac{1}{f_{r e s}}=2 l_{0} \cdot \sqrt{4 l_{0}^{2}+16 l_{0} v Z_{L} \cdot C} \\
& \text { with } C=C_{p}+C_{0, p} \cdot \varepsilon_{r, p}^{\prime}
\end{aligned}
$$

The half length of the resonator-ring is $l_{0}, v$ is the propagation speed of the wave, $Z_{L}$ is the impedance of the strip line, $C$ is the resulting capacitance [5]. Obviously a higher permittivity leads to a lower resonance frequency $f_{\text {res }}$.

To enable a continuious measurement as required for a detctor in LC, we apply an electromagnetic wave at constant frequency to the input port of the strip line and measure the amplitude at the output as a rectified direct current voltage using an envelope detector. For maximum sensitivity this electromagnetic wave should match the resonance frequency of the split-ring resonator when pure LC solvent is present at the split. If the resonance frequency is shifted in any direction the output voltage will rise.

\section{Experimental}

The measurements are performed with two different new split-ring resonator topologies and two different measuring principles (determination of the resonance frequency from the $S_{21}$ parameter measured with a vector network analyzer (VNA) vs. envelop detector at concstant input frequency). The first split-ring resonator is shown in Fig. 1. Its topology consists of a square ring with a large split capacitor between to strip lines directed to the middle of the ring. A simple sample container is placed on top of that split capacitor. 


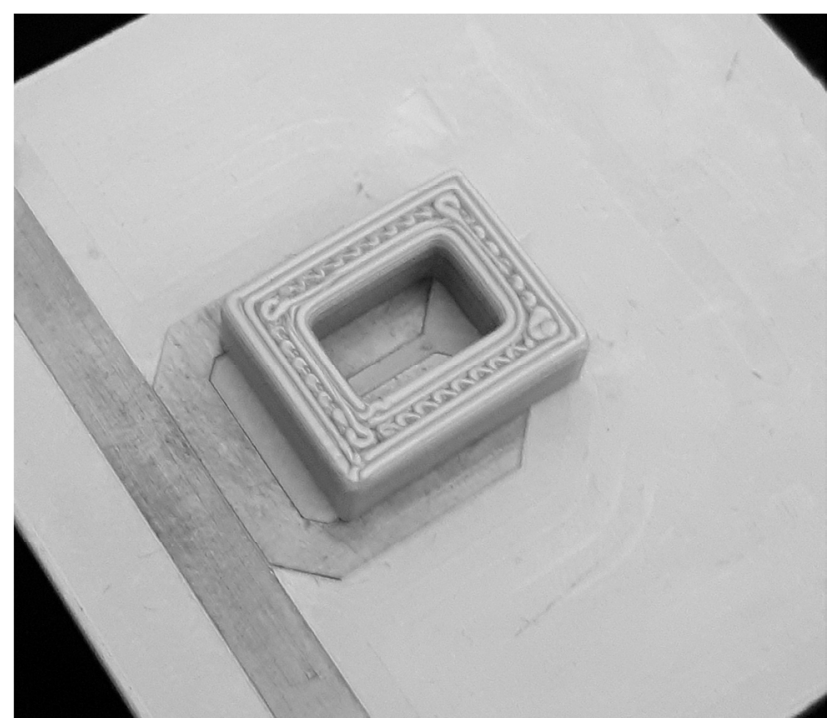

Fig. 1: Resonator (16 mm x $16 \mathrm{~mm}$ ) with sample container.

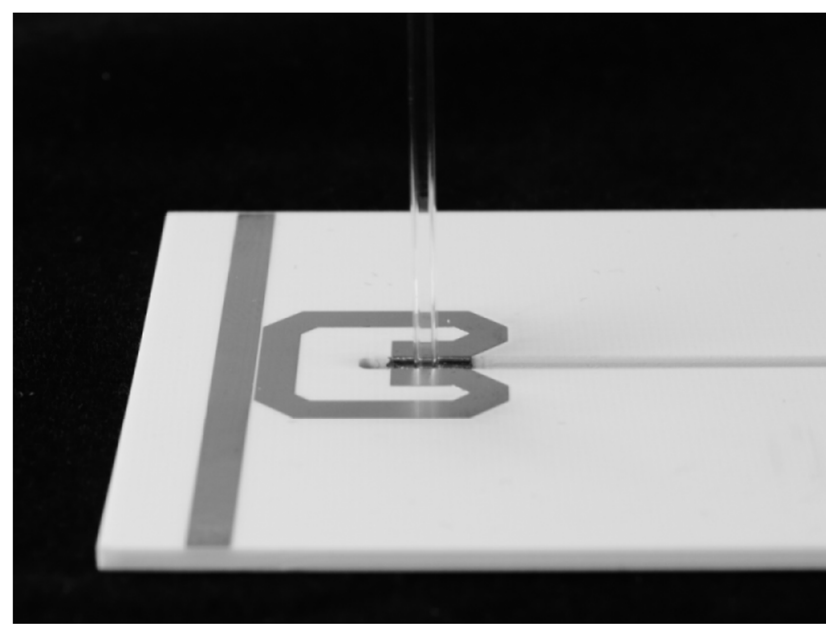

Fig. 2: Resonator (16 mm x $16 \mathrm{~mm}$ ) with flow through capillary.

The secod split-ring resonator has nearly the same topology but with a flow through capillary placed at the split capacitor to connect a LC system. Furthermore, the split capacitor is extended with sideplating. The split-ring resonator with flow through capillary is shown in Fig. 2. Due to the sideplating we reach sufficient electric field strength inside the capillary as seen in Fig. 3. Simulations in CST Microwave Studio also show the high sensitivity of this new split-ring resonator topology.

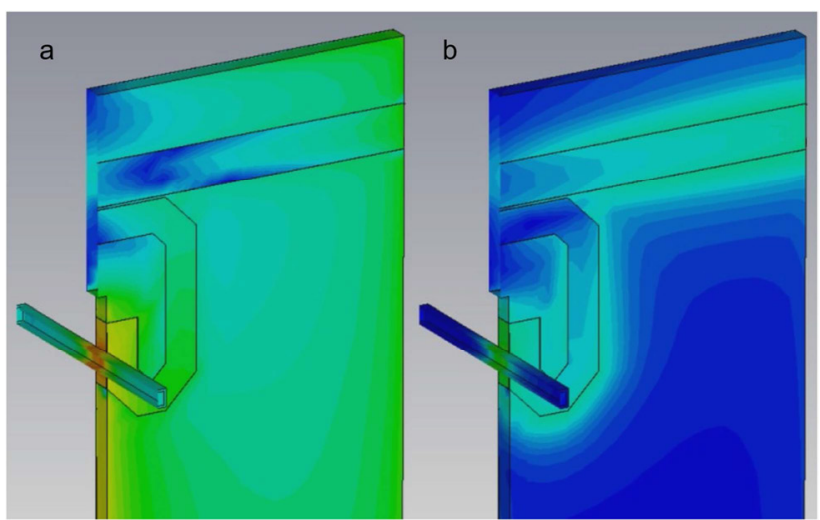

Fig. 3: Simulated electrical field; (a) in resonance; (b) not in resonance (red indicates high electric field strength, blue indicates low electric field strength).

As mentioned above, the measurements were performed with both a VNA to determine the resonance frequency from the $S_{21}$ parameter and a self-developed electronics (envelope detector at constant input frequence). To investigate the dependence of the resonance frequency on the permittivity of a sample deionized water-isopropanolmixtures and caffeine-deionized water solutions were used. The split-ring resonator is connected to the network analyzer via two SMA-ports that are connected to both ends of the strip line. To determine the resonance frequency the transmission is measured over a broad frequency range. Finally, the resonance frequency equals the frequency with lowest transmission. The frequency sweep has to be repeated for each sample. Thus, this principle suffers from long measurement times, not suitable for continously monitoring the resonsce frequency shift as required for any LC application. For continuous measurements, we use the rectified output of the split-ring resonator recorded with a self-developed envelope detector and constant input frequency. In resonance, the output is minimal because of the lowest transmission. The chosen fixed measuring frequency is the resonance frequency of the split-ring resonator when pure LC solvent (here, deionized water) is present. If another substance with different permittivity is present the rectified voltage output increases due to the decreasing coupling into the resonator following the shifted resonance frequency.

All measurements using the SRR with flow through capillary are performed with both measuring principles to investigate the shift of the resonance frequency and in order to prove the funcionality of the electronics for continuous measurements as required for LC. All measurements using the SRR with the sample container are performed with the VNA. 


\section{Results}

The calibration measurement using the network analyzer with mixtures of isopropanol and deionized water in the sample container on the split capacitor is shown in Fig. 4. The permittivity of each mixture is calculated as described by Åkerlöf [7].

As can be seen, the resonance frequency is highly dependent on the permittivity of the sample. The sensitivity varies with the operating point. It is between $4 \mathrm{MHz} / \Delta \varepsilon_{\mathrm{r}}$ and $56.4 \mathrm{MHz} / \Delta \varepsilon_{r}$. Considering the noise the system reaches a limit of detection of $0.126 \Delta \varepsilon_{r}$ at high relative permittivty around 78, $0.057 \Delta \varepsilon_{r}$ at medium relative permittivity around 45 and $0.009 \Delta \varepsilon_{r}$ at low relative permittivity around 13 .

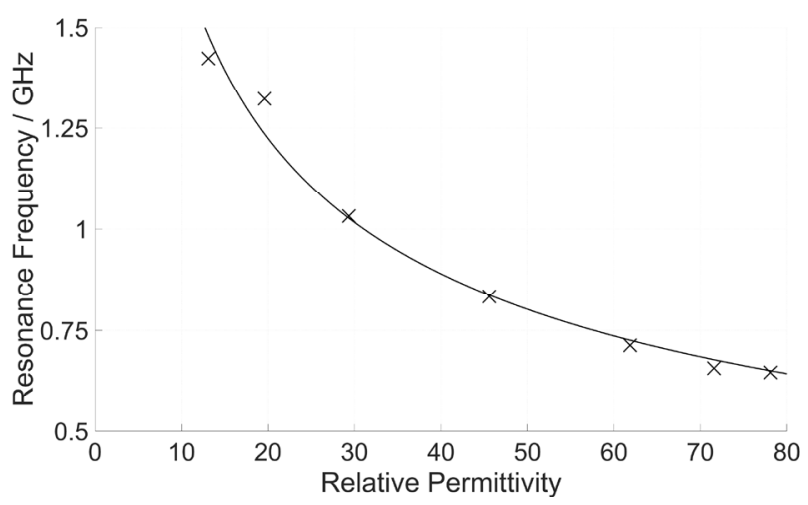

Fig. 4: Resonance frequency in dependence of the sample permittivity (SRR with sample container and VNA).

The detection of caffeine in aqueous solutions is a possible model application for LC. Therefore, the following measurement shows the dependence of the resonance frequency on the concentration of caffeine in deionized water, see Fig. 5. With increasing caffeine concentration the resonance frequency increases. This indicates that caffeine solutions have lower permittivity than pure deionized water as also reported by Lathi et al. They measured the dielectric constant of caffeine solutions up to $12 \mathrm{~g} / \mathrm{l}$ caffeine in deioized water. Their results show a moderate decreas of the permittivity from 78.1 for deionized water down to about 73 for $12 \mathrm{~g} / \mathrm{l}$ caffeine in deionized water at $1 \mathrm{GHz}$ [8].

Especially between 0 and $3 \mathrm{~g} / \mathrm{l}$ the resonance frequency shows a stronger increase. The sensitivity for low concentrations is about $1.023 \mathrm{MHz} / \Delta(\mathrm{g} / \mathrm{l})$. Considering the noise leads to a limit of detection of $0.49 \mathrm{~g} / \mathrm{l}$ for caffeine in deionized water. For comparison: The caffeine concentration of coffee is up to $1.33 \mathrm{~g} / \mathrm{l}$ [9].

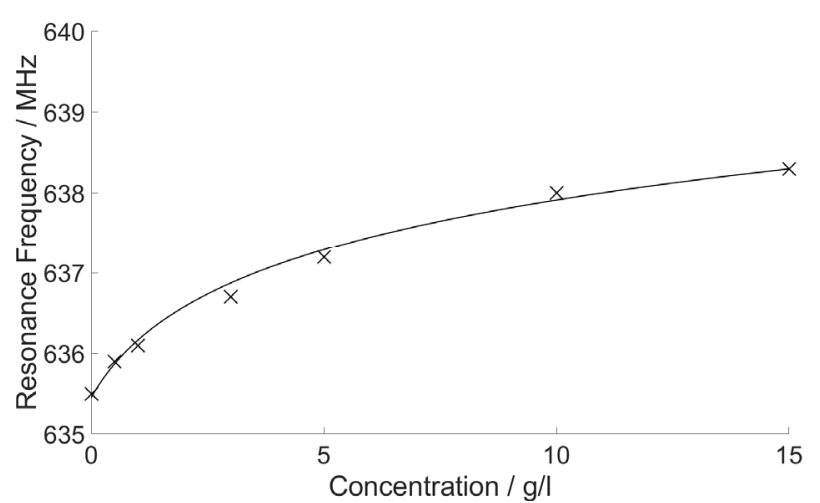

Fig. 5: Resonance frequency in dependence of the caffeine concentration in deionized water (SRR with sample container and VNA).

The calibration plot with mixtures of isopropanol and deionized water is also recorded with the SRR with the flow through capillary and VNA, see Fig. 6. Again, the resonance frequency decreases with increasing permittivity.

The resonance frequency decreases from $1.651 \mathrm{GHz}$ for pure isopropanol to $1.605 \mathrm{GHz}$ for pure deionized water. The sensitivity is between $0.3 \mathrm{MHz} / \Delta \varepsilon_{\mathrm{r}}$ and $1.9 \mathrm{MHz} / \Delta \varepsilon_{\mathrm{r}}$ depending on the operating point. In addition, the noise is reduced using a shielding enclosure. The system reaches a limit of detection between $0.107 \Delta \varepsilon_{\mathrm{r}}$ for low relative permittivity around 13 and $0.615 \Delta \varepsilon_{\mathrm{r}}$ for a relative permittivity around 78 .

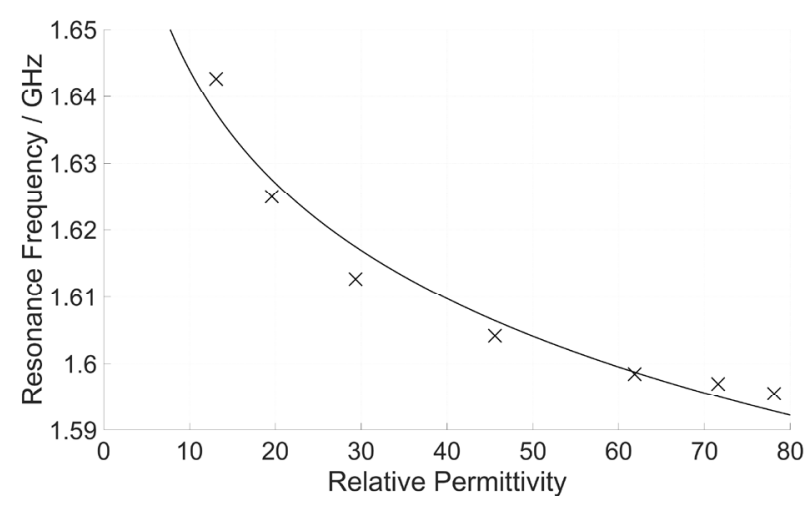

Fig. 6: Resonance frequency in dependence of the permittivity (SRR with flow through capillary and VNA).

Furthermore, the measurements with caffeine solutions are repeated with the SRR with flow through capillary and VNA, see Fig. 7. From pure deionized water to $3 \mathrm{~g} / \mathrm{l}$ a slight increase of the resonance frequency is visible. The sensitivity for low caffine concentrations is about $0.2 \mathrm{MHz} / \Delta(\mathrm{g} / \mathrm{l})$. Considering the noise leads to a limit of detection of $0.989 \mathrm{~g} / \mathrm{l}$ for caffeine in deionized water. Both measurements with the capillary and the VNA are obviously less sensitive than the 
measurement with the container and the VNA but the trend is similar. One reason is the significantly reduced interaction of the electrical field with the sample in the capillary due to field distortion caused by the capillary walls and the reduced sample volume in the spilt capacitor.

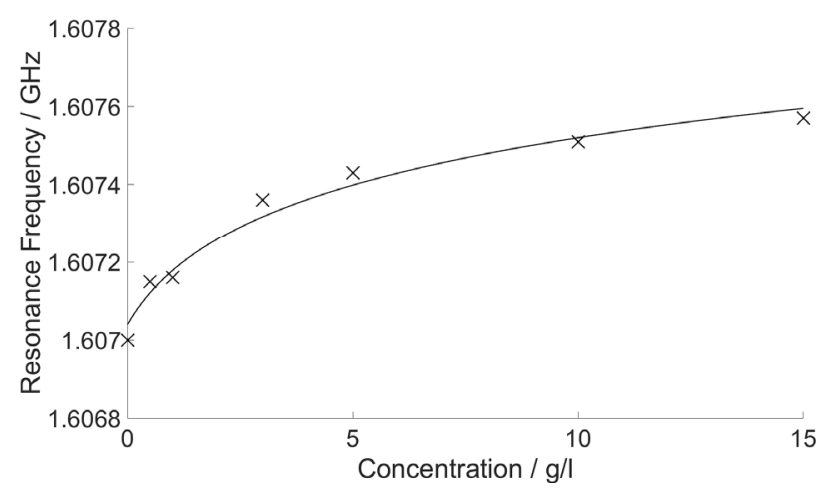

Fig. 7: Resonance frequency in dependence of the caffeine concentration in deionized water (SRR with flow through capillary and VNA).

To enable a continuous measurement the SRR with flow through capillary is connected to our selfdeveloped electronics and the measurements of isopropanol-deionized water-mixtures and caffeine solutions is repeated. The results are shown in Fig. 8. The output voltage decreases with increasing relative permittivity because the measuring frequency is set to the resonance frequency of deionized water that has a relative permittivity of 78.1. Therefore, the output voltage is at a minimum for this permittivity. With increasing permittivity, the measuring frequency does not match the resonance frequency and the output voltage increases. The sensitivity is $0.4 \mathrm{mV} / \Delta \varepsilon_{\mathrm{r}}$. Considering the noise the system reaches a limit of detection of $0.0375 \Delta \varepsilon_{r}$ for a averaging time of $100 \mathrm{~ms}$.

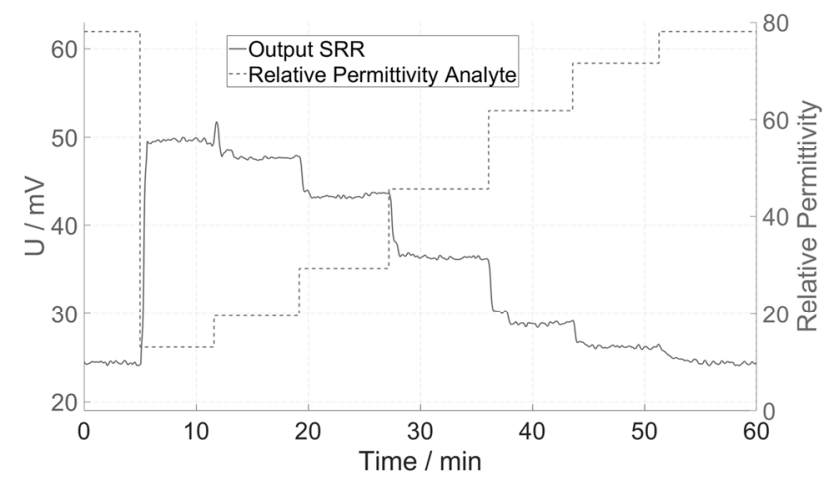

Fig. 8: Output in dependence of the permittivity (SRR with flow through capillary and envelope detector).

\section{Conclusion}

This work shows preliminary results using a new topology of a split-ring resonator and a new selfdeveloped electronics suitable for continuous detection of permittivity changes e.g. in a flow through capillary. While the sensitivity of the splitring resonator with a sample container is very high, using a flow through capillary significantly reduces the interaction of the electrical field with the sample due to field distortion caused by the capillary walls and the reduced sample volume in the spilt capacitor. Nevertheless with both split-ring resonators a detection of permittivity changes is possible. However, the signal-to-noise ratio using a flow through capillary in combination with the envelope detector is way off any LC application so that a new fluidic concept needs to be developed that allows maximum interaction between the electrical field and the sample but avoids any contact of the sample with the substrate material of the SSR causing memory effects due to sample uptake of the substrate material.

\section{References}

[1] O. Shakoor, R. B. Taylor, R. H. Behrens, Assessment of the incidence of substandard drugs in developing countries, Tropical Medicine and International Health 2, 839-845 (1997); doi: 10.1046/j.13653156.1997.d01-403.x.

[2] V. Di Stefano, G. Avellone, D. Bongiorno, V. Cunsolo, V. Muccilli, S. Sforza, A. Dossena, L. Drahos, K. Vékey, Applications of liquid chromatography-mass spectrometry for food analysis, Journal of chromatography. A 1259, 74-85 (2012); doi: 10.1016/j.chroma.2012.04.023.

[3] M. Swartz, HPLC Detectors: A Brief Review, Journal of Liquid Chromatography \& Related Technologies 33, 1130-1150 (2010); doi: 10.1080/10826076.2010.484356.

[4] A. Gehl, S. Zimmermann, Ein neues Detektorkonzept für die Flüssigchromatographie basierend auf einem Split-Ring-Resonator / A new detector concept for liquid chromatography based on a split-ring resonator, tm - Technisches Messen 85, s33-s37 (2018); doi: 10.1515/teme-2018-0032.

[5] T. Reinecke, J.-G. Walter, T. Kobelt, A. Ahrens, T. Scheper, S. Zimmermann, Design and evaluation of split-ring resonators for aptamer-based biosensors, Journal of Sensors and Sensor Systems 7, 101-111 (2018); doi: 10.5194/jsss-7-101-2018. 
[6] A. Verma, S. Bhushan, P. N. Tripathi, M. Goswami, B. R. Singh, A defected ground split ring resonator for an ultra-fast, selective sensing of glucose content in blood plasma, Journal of Electromagnetic Waves and Applications 31, 1049-1061 (2017); doi: 10.1080/09205071.2017.1325011.

[7] G. Åkerlöf, Dielectric Constants of some Organic Solvent-Water Mixtures at Various Temperatures, Journal of the American Chemical Society 54, 41254139 (1932); doi: 10.1021/ja01350a001.

[8] A. R. Lathi, S. K. Popalghat, Dielectric Relaxation Study of Alkaloid (Caffeine) in Aqueous Solution Using Time Domain Reflectrometry Technique, International Journal of Science and Research 4, 2409-2412 (2015).

[9] J. L. Blauch, S. M. Tarka, HPLC Determination of Caffeine and Theobromine in Coffee, Tea, and Instant Hot Cocoa Mixes, Journal of Food Science 48, 745-747 (1983). 